\title{
RNF213 as the major susceptibility gene for Chinese patients with moyamoya disease and its clinical relevance
}

\author{
*Qian Zhang, MD, ${ }^{1}$ Yaping Liu, PhD, ${ }^{2}$ Dong Zhang, MD, ${ }^{1}$ Rong Wang, MD, ${ }^{1}$ Yan Zhang, MD, ${ }^{1}$ \\ Shuo Wang, MD, Lanbing Yu, MD, ${ }^{1}$ Chaoxia Lu, PhD, ${ }^{2}$ Fang Liu, MS, ${ }^{2}$ Jian Zhou, MS, ${ }^{2}$ \\ Xue Zhang, PhD, MD, ${ }^{2}$ and Jizong Zhao, MD'
}

\begin{abstract}
1Department of Neurosurgery, Beijing Tiantan Hospital, Capital Medical University, China National Clinical Research Center for Neurological Diseases; and ${ }^{2}$ McKusick-Zhang Center for Genetic Medicine, State Key Laboratory of Medical Molecular Biology, Institute of Basic Medical Sciences, Chinese Academy of Medical Sciences and Peking Union Medical College, Beijing, People's Republic of China
\end{abstract}

\begin{abstract}
OBJECTIVE Moyamoya disease (MMD) is a rare, genetically heterogeneous cerebrovascular disease. The authors conducted a genetic study of really interesting new gene (RING) finger protein 213 (RNF213); actin alpha 2 (ACTA2); BRCA1/BRCA2-containing complex subunit 3 (BRCC3); and guanylate cyclase 1, soluble, alpha 3 (GUCY1A3) as well as a clinical phenotype analysis in Chinese MMD patients to determine whether genetic differences are responsible for the different clinical features that appear in MMD in different ethnicities.
\end{abstract}

METHODS A panel was designed to identify disease-causing mutations in MMD genes and those involved in related disorders (RNF213, ACTA2, BRCC3, and GUCY1A3). The panel was used to detect disease-causing mutations in 255 Chinese MMD patients. Genotype and allele frequencies were compared between patients and 300 controls. A mutation segregation analysis was performed in 34 families, and genotype-phenotype correlations were made.

RESULTS Twenty-seven rare missense variants of RNF213 were identified and were not found in controls. Among them, p.R4810K was identified in $31.4 \%$ of patients ( 80 of 255 ) with MMD. Significantly higher frequencies of the A allele and G/A genotype of $p . R 4810 \mathrm{~K}$ were observed in MMD patients compared with controls $\left(\chi^{2}=104.166, p<0.000\right)$. Twenty-five rare variants were identified in $10.6 \%$ of patients ( 27 of 255 ) without $p . R 4810 \mathrm{~K}$ variants. Segregation analysis supported an association between MMD and 3 variants. No possible disease-causing mutations were identified in ACTA2, $B R C C 3$, or GUCY1A3. Compared with patients without the rare variants in RNF213, the p.R4810K heterozygous patients were younger at diagnosis ( 25 vs 29 years old, $p=0.049$ ) and had more familial cases $(24 \%$ vs $4.4 \%, p=0.000$ ), ischemic cases $(81.3 \%$ vs $67.5 \%, p=0.037)$, and involvement of the posterior cerebral artery $(52 \%$ vs $32.5 \%, p=0.007)$.

CONCLUSIONS RNF213 is the major susceptibility gene in Chinese MMD patients. The spectrum of rare variants identified in Chinese MMD patients was diverse. Compared to patients without the rare variants in RNF213, the p.R4810K heterozygous patients exhibited different clinical features.

https://thejns.org/doi/abs/10.3171/2016.2.JNS152173

KEY WORDS moyamoya disease; RNF213; variant; clinical features; vascular disorders

$\mathrm{M}$ OYAMOYa disease (MMD) is a vasculopathy characterized by progressive stenosis of the internal carotid arteries and its proximal branches accompanied by the development of a compensatory collateral vessel network. ${ }^{27}$ Despite substantial investigation, the molecular etiology and pathogenesis of moyamoya angi- opathy remain unclear. Several studies have explored genetic factors, and 4 loci associated with MMD have been reported: 3p24-p26, ${ }^{10} 6 \mathrm{q} 25,{ }^{11} 8 \mathrm{q} 23,{ }^{25}$ and $17 \mathrm{q} 25 .{ }^{13,18} \mathrm{Mu}-$ tations in really interesting new gene (RING) finger protein 213 (RNF213); ${ }^{13,18}$ actin alpha 2 (ACTA2); ${ }^{7}$ BRCA1/ BRCA2-containing complex, subunit 3 (BRCC3); ${ }^{22}$ and

ABBREVIATIONS MAF = minor allele frequency; MMD = moyamoya disease; $M M S=$ moyamoya syndrome; $\mathrm{mRS}=$ modified Rankin Scale; NGS = next-generation sequencing; $P C A=$ posterior cerebral artery; RING = really interesting new gene.

SUBMITTED September 22, 2015. ACCEPTED February 9, 2016.

INCLUDE WHEN CITING Published online April 29, 2016; DOI: 10.3171/2016.2.JNS152173.

* Drs. Q. Zhang and Y. Liu contributed equally to this work. 
guanylate cyclase 1 , soluble, alpha $3(G U C Y 1 A 3)^{8}$ have been reported in patients with MMD or moyamoya syndrome (MMS).

$R N F 213$ is located at $17 \mathrm{q} 25$ and encodes a RING finger protein, a single protein that possesses both ubiquitin ligase activity and ATPase activity..$^{18}$ As reported in previous studies, there are differences in the spectrum of mutations between East Asians and whites. For example, p.C3997Y, p.D4013N, and p.R4019C variants in RNF213 have been identified only in white MMD patients,, 318 who did not exhibit p.R4810K, a highly recurrent variant in Asian patients. In East Asia, the attributable risks of a p.R4810K mutation in Japanese (145 [90\%] of 161 cases) and Korean (30 [79\%] of 38 cases) populations were significantly larger than in the Chinese population (12 [23\%] of 52 cases).$^{18}$ Moreover, patients with heterozygous ACTA 2 mutations have been reported to be predisposed to a variety of diffuse and diverse vascular diseases, including thoracic aortic aneurysms and dissections, ischemic stroke, and MMD. ${ }^{7} \mathrm{Xq} 28$ deletions removing mature T-cell proliferation 1/mature T-cell proliferation 1 neighbor and $B R C C 3$ may lead to moyamoya angiopathy, short stature, and a stereotyped facial dysmorphism. ${ }^{22}$ An autosomalrecessive disease associated with moyamoya and achalasia was identified recently, and it was caused by loss-of-function mutations in GUCY1A3. ${ }^{8}$ Whether ACTA2, BRCC3, and GUCY1A3 are causative genes of MMD in Chinese patients remains unknown. To date, there are no largescale genetic studies including Chinese MMD patients and families.

Moyamoya disease occurs worldwide, but its prevalence is highest in East Asian countries including Japan (6.03 cases/100,000 persons), Korea (6.3 cases/100,000 persons), and China (3.92 cases/100,000 persons). ${ }^{1,5,9,17,21}$ Its incidence in Europe and America appears to be approximately one-tenth of that in Japan. ${ }^{26}$ In China, a female predominance is less apparent than those in Japan and Western countries. ${ }^{4,15,30}$ Moreover, symptoms at the onset of MMD in China are different from those in Japan and South Korea but similar to those in the United States and Europe. ${ }^{4}$ The epidemiological and clinical characteristics of Chinese MMD are also different from those of other ethnicities of MMD. ${ }^{4}$ Whether genetic differences are responsible for the different clinical features remains unclear. We conducted a comprehensive genetic study of RNF213, ACTA2, BRCC3, and GUCY1A3 and a clinical phenotype analysis of Chinese MMD patients to address this question.

\section{Methods}

\section{Study Population and Sample Collection}

We recruited 255 MMD patients and 300 healthy controls at Beijing Tiantan Hospital, Capital Medical University, from June 2012 to October 2014. The MMD patients included 107 men and 148 women, with a mean age at participation of $26.7 \pm 14.7$ years (mean \pm standard deviation). The 255 cases consisted of 220 sporadic cases with no family history and 35 familial MMD cases from 16 families ( $>1$ family member with MMD). The other members of these 16 families and the parents of 18 childhood-onset patients out of the 220 sporadic MMD cases were also included in the study. Our 300 controls, 128 men and 172 women, consisted of age- and sex-matched healthy individuals who were seen for routine laboratory tests (mean age at participation $28.0 \pm 15.9$ years). All subjects were Han Chinese.

Diagnosis of MMD was based on MR angiography and/or diagnostic angiography findings demonstrating stenosis or occlusion of the terminal portion of the internal carotid artery with the formation of collateral vessels compensating for the arterial occlusion. ${ }^{24}$ Patients diagnosed with both unilateral and bilateral MMD were included in this study. Other causes of arterial occlusion, such as atherosclerosis, meningitis, Down syndrome, or systemic vasculitis, were excluded via medical record and imaging review. Information on sex, age at diagnosis, family history, onset of symptoms, and preoperative modified Rankin Scale (mRS) score were obtained through clinical chart review. Angiography profiles, including combined aneurysm, stenoocclusive lesions of the posterior cerebral artery (PCA), dilation of the anterior choroidal artery, and Suzuki stage, were classified by 2 neurosurgeons. Blood samples were collected from the samples mentioned above after written informed consent had been obtained. The Ethics Committee of Beijing Tiantan Hospital, Capital Medical University, approved the study.

\section{DNA Sequencing and Data Analysis}

Genomic DNA from the 255 patients was extracted from blood leukocytes using a QIAamp blood kit (Qiagen) and was sequenced with next-generation sequencing (NGS) using a custom-designed Ion AmpliSeq Panel (Life Technologies) and Ion Torrent personal genome machine (PGM) ${ }^{28}$ As key words, "moyamoya disease" was searched in Online Mendelian Inheritance in Man (OMIN). RNF213 (moyamoya disease 2), ACTA2 (moyamoya disease 5), BRCC3 (moyamoya disease 4), and GUCY1A3 (moyamoya disease 6) had been reported as susceptibility or causative genes in MMD or MMS patients and were selected as target genes. A panel was designed to identify disease-causing mutations in the exons and exon-intron boundaries ( $\pm 50 \mathrm{bp}$ ) of these genes. The descriptions of these genes (RNF213 ACTA2, BRCC3, and $G U C Y 1 A 3)$ in this paper are primarily based on the longest isoforms (NM_001256071, NM_001141945, NM_024332, and NM_000856, respectively). Exon sequencing was conducted according to the manufacturer's protocol. The details are shown in the Supplemental Materials and Methods.

Software, including Torrent Suite 4.0 (Life Technologies), SeattleSeq Annotation version 9.00 (University of Washington), and Integrative Genomics Viewer 2.1 (IGV, Broad Institute), was used to perform bioinformatics analyses such as optimizing signal processing, base calling, sequence alignment, and variant analysis. Gene variants identified via exome sequencing were filtered. Only variants with a minor allele frequency $(\mathrm{MAF})<$ $5 \%$ in the Exome Variant Server database (http://evs. gs.washington.edu/EVS/) and the 1000 Genomes database (http://1000genomes.org) were retained for further investigation. Finally, identified rare variants (arbitrarily defined 
as MAF $<5 \%$ in this study) were confirmed by Sanger sequencing using an ABI 3730 DNA analyzer (Applied Biosystems). The details of the primers used for amplification are shown in Supplemental Table 1. Potential effects of amino acid substitutions were predicted by PolyPhen-2 (http://genetics.bwh.harvard.edu/pph2/) and SIFT (http:// sift.jcvi.org/).

\section{Clinical Phenotype Analysis in Different Genotypes}

We assessed the clinical and angiographic features according to genotypes, including sex ratio, age at diagnosis, family history, frequency of childhood onset, clinical manifestations, preoperative mRS score $>2$, combined aneurysm, PCA involvement, dilation of anterior choroidal artery, and Suzuki stage.

\section{Statistical Analysis}

All statistical analyses were performed using SPSS Statistics version 19 software (IBM Corp.). Differences in genotype and allele frequencies between patients and controls were compared. Differences in allele frequency were quantified by odds ratios and $95 \%$ confidence intervals. A chi-square distribution was used to analyze associations between categorical clinical characteristics and genotypes. Nonnormally distributed continuous variables, such as age at diagnosis and Suzuki stage, were compared using the Mann-Whitney U-test between different genotypes. Statistical significance was defined as 2 -tailed $\mathrm{p}<0.05$.

\section{Results \\ DNA Sequencing Results}

Two hundred fifty-five MMD patients were sequenced with NGS using a custom-designed Ion AmpliSeq Panel. The mean depth was more than 150 times (that is, each base was sequenced 150 times), and the on-target rate exceeded $95 \%$. There were no possible disease-causing mutations identified in ACTA2, BRCC3, and GUCY1A3. Thirty-seven rare missense variants of $R N F 213,16$ of which were novel, were identified. No frame-shift or nonsense mutations were detected. These variants were p.P61L, p.A1041T, p.A1041V, p.R1228G, p.E1707K, p.T1727M, p.T1866I, p.P2107L, p.N2971Y, p.E3061K, p.G3470R, p.R3580W, p.M3666T, p.I3693L, p.D3899N, p.G3936E, p.D4013N, p.H4014T, p.A4050V, p.R4062Q, p.N4066S, p.K4160Q, p.P4250T, p.M4289I, p.S4389G, p.A4399T, p.P4576S, p.G4640R, p.R4810K, p.D4863N, p.V4884I, p.Q4938R, p.E4950D, p.S5012R, p.A5021V, p.T5037I, and p.R5153H and were confirmed by Sanger sequencing (Table 1 and Supplemental Table 2).

\section{Comparison of the Genotype and Allele Frequencies Between Patients and Controls}

Among the 37 rare variants of $R N F 213,27$ were not found in the 300 healthy controls (Fig. 1; Supplemental Table 3). The MAF values of these 27 mutations were less than $0.08 \%$ in the Exome Variant Server database and the 1000 Genomes database. Among these mutations, p.R4810K was identified in $31.4 \%$ of patients ( 80 of 255) with MMD (65.7\% familial cases and $25.9 \%$ nonfamil- ial patients). The p.T1727M variant was identified only in patients with the p.R4810K mutation (29 [36.3\%] of 80). Significantly higher frequencies of the A allele and G/A genotype of p.R4810K (rs112735431) and the T allele and T/C genotype of p.T1727M (rs371978343) were observed in MMD patients compared with controls $\left(\chi^{2}=104.166\right.$, $\mathrm{p}<0.000 ; \chi^{2}=35.033, \mathrm{p}<0.000$, respectively). The other 25 rare variants (not found in controls) were identified in $10.6 \%$ of patients (27 of 255 ) without p.R4810K variants. No significant association between MMD and any of the 25 rare mutations in $R N F 213$ was detected. In total, $42 \%$ of patients (107 of 255) carried the p.R4810K variants or the possible susceptibility variants in RNF213 (Table 1 and Fig. 1).

\section{Mutation Segregation Analysis}

Ten $(62.5 \%)$ of the 16 MMD families (> 1 family member with MMD) carried the p.R4810K variant in RNF213. It co-segregated with MMD and was not fully penetrant. A novel variant, p.R4160Q, was confirmed to co-segregate with MMD in 1 family, but it was not fully penetrant. A de novo p.H4014T variant in the RING finger domain of $R N F 213$ was detected in 1 family (Fig. 2).

\section{Comparison of Clinical Features Between Patients With the RNF213 p.R4810K Heterozygous Variant and Those Without Rare Variants in RNF213}

We analyzed the clinical features of patients with MMD according to the $R N F 213$ variants. Patients carrying the heterozygous p.R4810K variant were classified as Group 1 , and those carrying no RNF213 rare variants were classified as Group 2. Among the 255 MMD patients, 80 patients carried p.R4810K variants. Two homozygous patients and 3 patients without cerebral angiography were excluded; therefore, 75 cases were included in Group 1. Among the remaining 175 study entrants, 58 were excluded because they carried other $R N F 213$ rare variants mentioned above and 3 because they had no cerebral angiography; thus, 114 patients were enrolled in Group 2. Demographic data for the 189 patients are summarized in Table 2 . The female/ male ratio was $1.5: 1$. The mean age at diagnosis was 27.7 years (range 3-59 years). Of the 189 patients, 23 (12.2\%) had a familial history, and 61 (32.3\%) had a childhood onset $(<18$ years old). Ischemic symptoms were identified in 138 patients $(73.0 \%)$, and hemorrhage was observed in 51 (27.0\%). Ten intracranial aneurysms (5.3\%) were recorded. A preoperative $\mathrm{mRS}$ score $>2$ was observed in 26 patients (13.8\%). Angiographs from 76 patients (40.2\%) showed stenoocclusive lesions of the PCA. The angiographic stages of anterior circulation were as follows: Stage 1 in 4 hemispheres (1.0\%), Stage 2 in $111(29.4 \%)$, Stage 3 in $60(15.9 \%)$, Stage 4 in 135 (35.7\%), Stage 5 in 45 (11.9\%), and Stage 6 in 23 (6.1\%). Dilation of the anterior choroidal artery was observed in 192 hemispheres (50.8\%).

Compared with the patients without rare variants (MAF $<0.05)$ in RNF213, p.R4810K heterozygous patients were younger at diagnosis (25 vs 29 years old, $\mathrm{p}=0.049)$ and included more familial cases $(24 \%$ vs $4.4 \%, \mathrm{p}=0.000)$, more ischemic cases $(81.3 \%$ vs $67.5 \%, \mathrm{p}=0.037)$, and more stenoocclusive lesions in the PCA $(52 \%$ vs $32.5 \%$, 
TABLE 1. Rare variants of RNF213 (not found in controls) and the predicted effects of amino acid substitutions

\begin{tabular}{|c|c|c|c|c|c|c|}
\hline Amino Acid Change & Position (chr17) & rs ID & MAF in NHLBI ${ }^{*}$ & MAF in 1000 Genomest & PolyPhen-2 HumDiv & SIFT \\
\hline p.R1228G & 78305970 & - & 0 & 0 & Possibly D $\ddagger$ & $\mathrm{D} \ddagger$ \\
\hline p.E1707K & 78313286 & 540300391 & 0.0002 & 0 & Probably D $\ddagger$ & $\mathrm{D} \ddagger$ \\
\hline p.T1727M & 78313347 & 371978343 & 0 & 0.000219 & Benign & Tolerated \\
\hline p.P2107L & 78317793 & - & 0 & 0 & Probably $\mathrm{D} \ddagger$ & Tolerated \\
\hline p.N2971Y & 78321046 & - & 0 & 0 & Probably D $\ddagger$ & Tolerated \\
\hline p.E3061K & 78321316 & - & 0 & 0 & Benign & Tolerated \\
\hline p.G3470R & 78326844 & - & 0 & 0 & Benign & Tolerated \\
\hline p.R3580W & 78328252 & 554959669 & 0.0002 & 0 & Benign & $\mathrm{D} \ddagger$ \\
\hline p.13693L & 78333883 & - & 0 & 0 & Benign & $\mathrm{D} \ddagger$ \\
\hline p.D3899N & 78337535 & - & 0 & 0 & Possibly D $\ddagger$ & $\mathrm{D} \ddagger$ \\
\hline p.G3936E & 78338289 & - & 0 & 0 & Benign & Tolerated \\
\hline p.D4013N & 78341825 & 397514563 & 0 & 0 & Possibly D $\ddagger$ & Tolerated \\
\hline p.H4014T & 78341828 & - & 0 & 0 & Probably D $\ddagger$ & $\mathrm{D} \ddagger$ \\
\hline p.A4050V & 78341937 & - & 0 & 0 & Benign & Tolerated \\
\hline p.R4062Q & 78343331 & - & 0 & 0 & Probably D $\ddagger$ & $\mathrm{D} \ddagger$ \\
\hline p.N4066S & 78343343 & - & 0 & 0 & Probably D $\ddagger$ & $\mathrm{D} \ddagger$ \\
\hline p.K4160Q & 78345726 & - & 0 & 0 & Probably D $\ddagger$ & Tolerated \\
\hline p.M4289| & 78346890 & 374262786 & 0 & 0.00008 & Benign & Tolerated \\
\hline p.S4389G & 78349650 & 187389872 & 0.0002 & 0 & Benign & Tolerated \\
\hline p.G4640R & 78355467 & 138223459 & 0 & 0.00015 & Benign & Tolerated \\
\hline p.R4810K & 78358945 & 112735431 & 0.0012 & 0 & Possibly D $\ddagger$ & Tolerated \\
\hline p.V4884I & 78360160 & 146990608 & 0.0004 & 0.00008 & Possibly D $\ddagger$ & Tolerated \\
\hline p.Q4938R & 78360582 & - & 0 & 0 & Possibly D $\ddagger$ & $\mathrm{D} \ddagger$ \\
\hline p.E4950D & 78360619 & 371441113 & 0.0008 & 0 & Probably D $\ddagger$ & Tolerated \\
\hline p.S5012R & 78363006 & - & 0 & 0 & Benign & Tolerated \\
\hline p.T5037I & 78363082 & - & 0 & 0 & Possibly D $\ddagger$ & Tolerated \\
\hline p.R5153H & 78363984 & 528073196 & 0.0002 & 0 & Probably D $\ddagger$ & Tolerated \\
\hline
\end{tabular}

$\mathrm{p}=0.007)$. There was no significant difference in the sex ratio $(1.4: 1$ vs $1.8: 1, \mathrm{p}=0.707)$, frequency of childhood onset $(38.7 \%$ vs $28.1 \%, \mathrm{p}=0.127)$, preoperative $\mathrm{mRS}$ score $>2(9.3 \%$ vs $16.7 \%, \mathrm{p}=0.152)$, combined aneurysm $(1.3 \%$ vs $7.9 \%, \mathrm{p}=0.123$ ), dilation of anterior choroidal artery (56.0\% vs $47.4 \%, \mathrm{p}=0.101)$, or Suzuki stage $(\mathrm{p}=0.486)$ between the 2 groups.

\section{Discussion}

The results of this study indicate that RNF213 is the major susceptibility gene for Chinese MMD patients. Moreover, no disease-causing mutations were identified in $A C T A 2, B R C C 3$, or GUCY1A3. Compared with the rate in previous studies of Chinese MMD patients, a much higher frequency of the p.R4810K variant was observed in our study $(31.4 \%$ vs $9.4 \%-23 \%) .{ }^{18,29,30}$ We also showed a higher prevalence of the p.R4810K mutation in familial MMD cases $(65.7 \%)$ than in sporadic ones $(25.9 \%)$, which was in line with Japanese MMD cases. ${ }^{13,18}$ We noticed that 5 familial cases were enrolled in previous studies on Chinese patients with MMD. Thus, this difference may be due to the higher proportion of familial cases (35 [13.7\%] of 255) in our study. In East Asia, the attributable risks for the p.R4810K mutation in the Japanese (145 [90\%] of 161 cases) and Korean (30 [79\%] of 38 cases) populations were significantly greater than that in the Chinese population (12 [23\%] of 52 cases). ${ }^{18}$ It has been suggested that other rare variants of $R N F 213$ may be causative mutations for those patients carrying them. By direct sequencing all exons and introns of RNF213 in 40 Chinese MMD patients without a p.R4810K variant, 5 variants - p.D4863N, p.E4950D, p.A5021 V, p.D5160E, and p.E5176G-were identified in 7 cases. The 5 variants were not detected in 150 Chinese controls. ${ }^{18}$ However, these variants were not verified in a large-scale case-control study. In our study, 


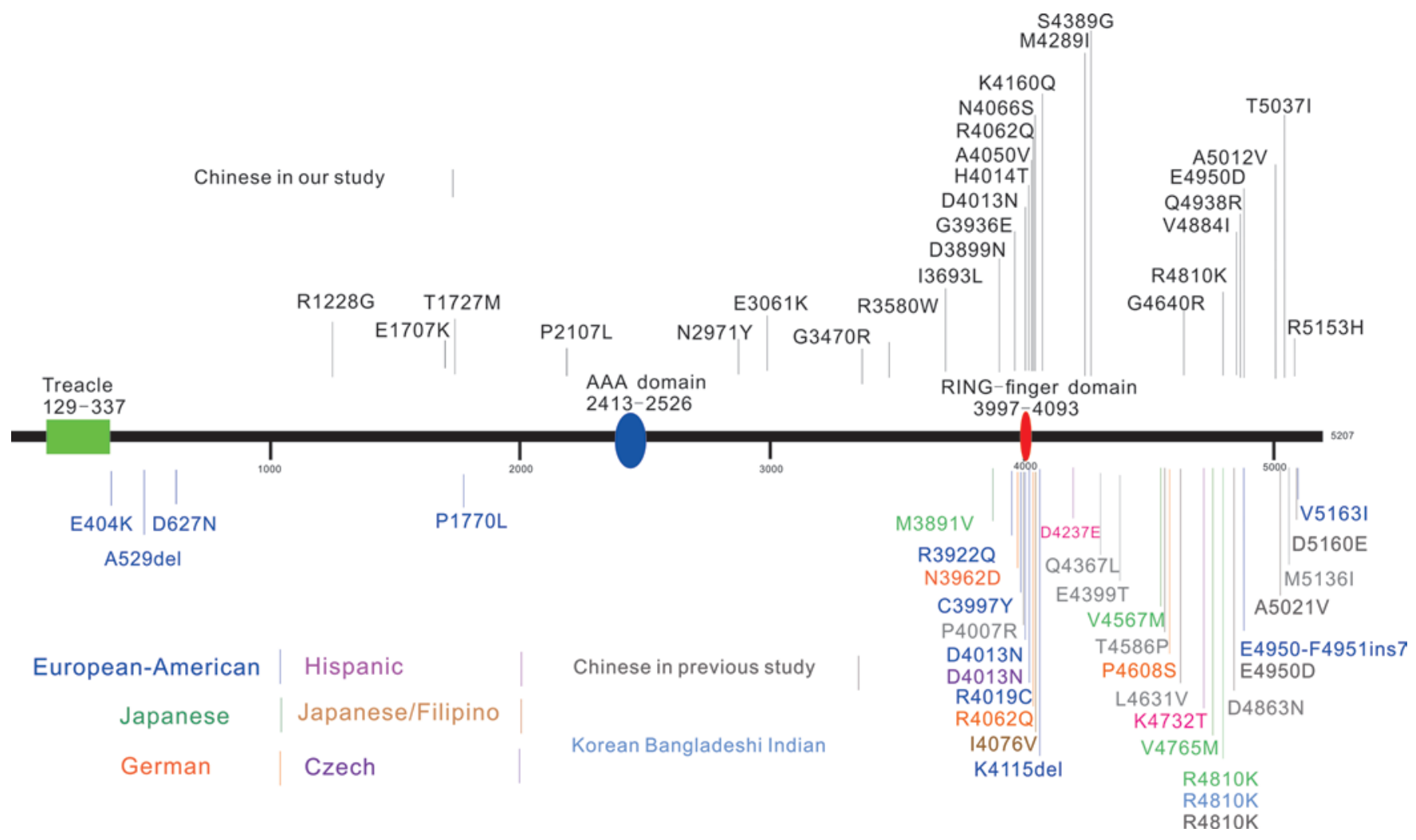

FIG. 1. Domains of RNF213 and variants identified in MMD. The 27 missense variants identified in Chinese patients with MMD in this study are shown in black typeface above the black line. The RNF213 rare variants identified in ethnically diverse populations in previous reports are shown below the black line. Different colors represent different ethnicities. Figure is available in color online only.

p.D4863N, p.E4950D, and p.A5021V were identified. Moreover, 16 novel variants were observed, and 27 rare variants (MAF < $0.08 \%)$ were not detected in controls. Segregation analysis supported an association between MMD and 2 novel variants (p.H4014T and p.R4160Q). We expanded the spectrum of rare variants in MMD patients. To date, most variants identified in RNF213 of MMD patients were missense variants. ${ }^{3,18,23}$ However, as the most widely reported variants, p.R4810K or p.D4013N did not affect ubiquitin ligase activity or cause other hallmark changes such as mRNA or protein instability in vitro. ${ }^{18}$ Further studies on the protein function of RNF213 may help to define the roles of these variants.

The spectrum of rare variants identified here supports the idea of diversity among ethnicities. The p.R4810K variant, a highly recurrent variant in Asian cases, was not found in white cases. ${ }^{3,18}$ Unlike p.R4810K, the variants of p.M3891V, p.V4567M, and p.V4765M were only identified in Japanese MMD cases. ${ }^{13}$ Other variants, such as A529del, p.R3922Q, p.C3997Y， R4019C， I4076V， K4115del, D4237E, K4732T, E4950_F4951ins7, and V5163I, have been identified only in white MMD patients. ${ }^{3}$ To date, several studies have reported different rare variants in different populations. ${ }^{3,6,13,18}$ The extensive allelic heterogeneity and ethnicity-specific variant spectrum of MMD were observed here (Fig. 1). It has been widely reported that the epidemiological and clinical characteristics of MMD vary in different ethnicities. ${ }^{1,4,5,9,21}$ However, the effect of genotype on clinical features is poorly understood. The homozygous c.14576G > A (rs112735431) variant carriers showed a significantly earlier age at onset and greater PCA involvement. ${ }^{23}$ However, no significant difference was observed between heterozygous and wild-type GG variants. Compared with a previous report, ${ }^{23}$ more adult (67.7\% vs $29.9 \%)$ and GG patients $(60.3 \%$ vs $17.6 \%)$ were included in our study. Moreover, in our Group 2 (GG carriers), patients with rare variants of $R N F 213$ were excluded because of their potential susceptibility to MMD. Thus, to some extent, patients in Group 2 could represent a series of MMD patients without any disease-causing mutations in RNF213, ACTA2, BRCC3, or GUCY1A3. We observed that fewer familial cases were found in Group $2(\mathrm{p}<0.05)$. Because of the complex nature of the disease's pathogenicity, it was reasonable to postulate that environmental factors, recessive inheritance, dominant inheritance with lower penetrance, or other polygenic inheritances were the possible pathogenic mechanisms in those patients. Recently, a genetic study of 170 Chinese patients with MMD showed the p.A4399T variation to be a potential risk factor for MMD, especially the hemorrhage subtype..$^{30}$ In our study, approximately $20 \%$ of adult MMD patients presented with intracranial hemorrhage. The variant p.A4399T, 


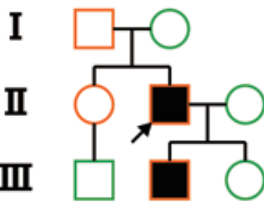

F1

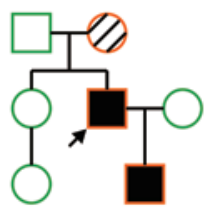

F4

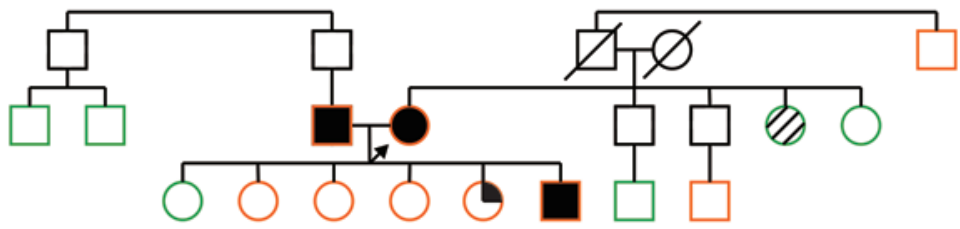

F3

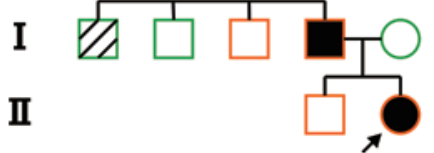

F2

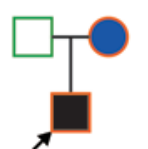

F5

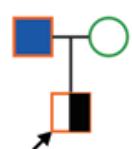

F11

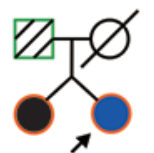

F12

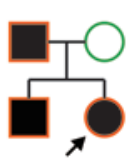

F13

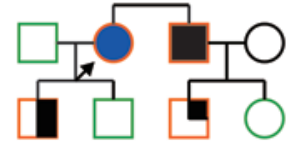

F14

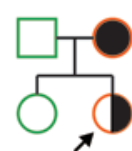

F16

p.R4160Q p.R3580W p.H4014T p.P4567S p.A1041V

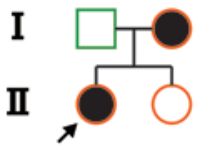

F15

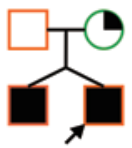

F6

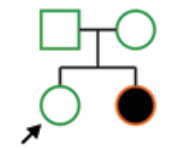

F17

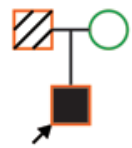

F21

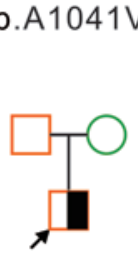

F24

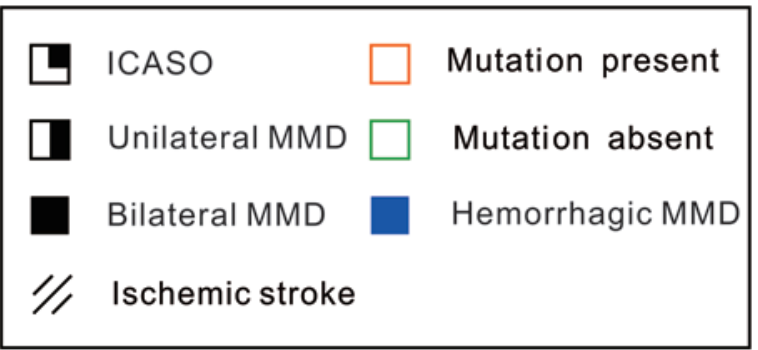

FIG. 2. Co-segregation of rare variants in RNF213 with MMD in families. Pedigrees of 10 families (> 1 family member with MMD, upper and center rows) with the RNF213 p.R4810K variant (F1-F5, F11-F14, and F16). The p.R4810K variant co-segregates with intracranial major artery stenosis/occlusion (ICASO), unilateral MMD, bilateral MMD, hemorrhagic MMD, and ischemic stroke. Pedigrees of 5 families (lower row) with other RNF213 rare variants. The p.R4160Q alteration co-segregates with MMD in F15. The p.R3580W variant does not co-segregate with MMD in F6. A de novo variant, p.H4014T, was identified in F17. Disease and mutation status are indicated in the figure key. Circles represent women, squares represent men, and arrowheads indicate the proband in the family. A diagonal line through a circle or square indicates that the individual is deceased. Figure is available in color online only.

which is a common one, is not a risk factor for MMD and hemorrhage. However, patients without rare variants detected in RNF213 were prone to exhibit hemorrhage. Although cerebral hemorrhage is mainly due to rupture of the fragile, deep, collateral vessels and aneurysms, ${ }^{14}$ the presence of a combining aneurysm or the dilation of an anterior choroidal artery was not significantly different between the 2 groups. There was also no significant difference in the Suzuki stages between the 2 groups. However, Suzuki Stage 3, which is the classic angiographic finding of MMD, is more often observed in p.R4810K heterozygotes ( $\mathrm{p}<0.05)$. Mice in which RNF213 was knocked out show enhanced postischemic angiogenesis. ${ }^{12}$ This finding suggests a potential role for the RNF213 malfunction in the development of pathological vascular networks. Our data suggest that MMD patients with the p.R4810K variant may have a distinct cerebrovascular disease entity and may represent a separate subgroup.

Moyamoya disease is a progressive disorder. Its natural history varies from slow progression to rapid neurological decline. ${ }^{16,19,26}$ Preventing strokes and the resulting comorbidities depends on the early identification of individuals who are predisposed to MMD and MMS. Early diagnosis allows for timely surgical intervention to reduce the risk of stroke and possibly decrease the resulting cognitive deficits. ${ }^{19}$ The NGS platform used in this study has great potential for clinical application. ${ }^{2,20,28}$ Screening for rare variants of RNF213 should be considered for patients with MMD. In addition, categorization of the clinical phenotypes and advances in NGS tools will undoubtedly accelerate the identification of novel familial MMD and MMS variants and patients. ${ }^{20}$

Our study is limited by its relatively small sample size and the absence of both protein functional tests and more detailed clinical data on the MMD patients.

\section{Conclusions}

$R N F 213$ is the major susceptibility gene in Chinese MMD patients. The spectrum of rare variants identified in Chinese MMD patients underscores the ethnic diversity in the variants found in this disease. Finally, compared to patients without the rare variants in $R N F 213$, p.R $4810 \mathrm{~K}$ heterozygous patients exhibited different clinical features.

\section{Acknowledgments}

We are grateful to the patients and their families for their participation in this study. This work was supported by Grant Nos. 
TABLE 2. Clinical features of patients with the p.R4810K heterozygous variant and those without rare and novel variants in RNF213

\begin{tabular}{|c|c|c|c|c|c|}
\hline Feature & Total & $\begin{array}{c}\text { Group 1, w/ p.R4810K } \\
\text { Heterozygous Variant (\%) }\end{array}$ & $\begin{array}{c}\text { Group 2, w/o RNF213 Rare } \\
\text { \& Novel Variants (\%) }\end{array}$ & $\chi^{2}$ or $Z$ & $\mathrm{p}$ Value \\
\hline No. of patients* & 189 & 75 & 114 & & \\
\hline Sex (F/M) & $1.5: 1$ & $1.4: 1$ & $1.8: 1$ & 0.142 & 0.707 \\
\hline No. of females (\%) & $114(60.3)$ & $44(58.7)$ & $70(61.4)$ & - & - \\
\hline Mean age at diagnosis in yrs $†$ & $27.7 \pm 14.6$ & $25.2 \pm 14.6$ & $29.3 \pm 14.4$ & $-1.971 \ddagger$ & 0.049 \\
\hline w/ familial history $(\%)$ & $23(12.2)$ & $18(24)$ & $5(4.4)$ & 16.283 & 0.000 \\
\hline Frequency of childhood onset (\%)§ & $61(32.3)$ & $29(38.7)$ & $32(28.1)$ & 2.324 & 0.127 \\
\hline \multicolumn{6}{|l|}{ Symptoms (\%) } \\
\hline Ischemia & $138(73.0)$ & $61(81.3)$ & $77(67.5)$ & 4.366 & 0.037 \\
\hline Hemorrhage & $51(27.0)$ & $14(18.7)$ & $37(32.5)$ & 4.366 & 0.037 \\
\hline Combined aneurysm & $10(5.3)$ & $1(1.3)$ & $9(7.9)$ & 2.378 & 0.123 \\
\hline Preop mRS score >2 & $26(13.8)$ & $7(9.3)$ & $19(16.7)$ & 2.051 & 0.152 \\
\hline PCA involvement & $76(40.2)$ & $39(52)$ & $37(32.5)$ & 7.187 & 0.007 \\
\hline Suzuki stage, 378 hemispheres (\%) & 378 & 150 & 228 & $-0.696 \ddagger$ & 0.486 \\
\hline 1 & $4(1.0)$ & $1(0.67)$ & $3(1.3)$ & 0.008 & 0.929 \\
\hline 2 & $111(29.4)$ & $37(24.7)$ & $74(32.5)$ & 2.647 & 0.104 \\
\hline 3 & $60(15.9)$ & $33(22.0)$ & $27(11.8)$ & 6.991 & 0.008 \\
\hline 4 & $135(35.7)$ & $51(34.0)$ & $84(36.8)$ & 0.318 & 0.573 \\
\hline 5 & $45(11.9)$ & $19(12.67)$ & $26(11.4)$ & 0.138 & 0.711 \\
\hline 6 & $23(6.1)$ & $9(6.0)$ & $14(6.1)$ & 0.003 & 0.955 \\
\hline Dilation of AChA, 378 hemispheres (\%) & $192(50.8)$ & $84(56.0)$ & $108(47.4)$ & 2.697 & 0.101 \\
\hline
\end{tabular}

$-=$ not applicable; $\mathrm{AChA}=$ anterior choroidal artery.

* Five (2 homozygous patients and 3 patients without cerebral angiography) of 80 patients in Group 1 and 58 (58 patients with other RNF213 rare variants and 3 patients without cerebral angiography) of 175 patients in Group 2 were excluded.

$\dagger$ Mean \pm standard deviation.

$\ddagger Z$ value.

$\S$ Onset at age $<18$ years.

2010DFB30850 (J. Zhao) and Z13110200680000 (D.Z.) from the Beijing Municipal Science and Technology Commission, China.

\section{References}

1. Ahn IM, Park DH, Hann HJ, Kim KH, Kim HJ, Ahn HS: Incidence, prevalence, and survival of moyamoya disease in Korea: a nationwide, population-based study. Stroke 45:1090-1095, 2014

2. Campens L, Callewaert B, Muiño Mosquera L, Renard M, Symoens S, De Paepe A, et al: Gene panel sequencing in heritable thoracic aortic disorders and related entities-results of comprehensive testing in a cohort of 264 patients. Orphanet J Rare Dis 10:9, 2015

3. Cecchi AC, Guo D, Ren Z, Flynn K, Santos-Cortez RL, Leal SM, et al: $R N F 213$ rare variants in an ethnically diverse population with Moyamoya disease. Stroke 45:3200-3207, 2014

4. Duan L, Bao XY, Yang WZ, Shi WC, Li DS, Zhang ZS, et al: Moyamoya disease in China: its clinical features and outcomes. Stroke 43:56-60, 2012

5. Gross BA, Du R: The natural history of moyamoya in a North American adult cohort. J Clin Neurosci 20:44-48, 2013

6. Guey S, Tournier-Lasserve E, Hervé D, Kossorotoff M: Moyamoya disease and syndromes: from genetics to clinical management. Appl Clin Genet 8:49-68, 2015

7. Guo DC, Papke CL, Tran-Fadulu V, Regalado ES, Avidan N, Johnson RJ, et al: Mutations in smooth muscle alpha-actin
(ACTA2) cause coronary artery disease, stroke, and Moyamoya disease, along with thoracic aortic disease. Am J Hum Genet 84:617-627, 2009

8. Hervé D, Philippi A, Belbouab R, Zerah M, Chabrier S, Collardeau-Frachon S, et al: Loss of $\alpha 1 \beta 1$ soluble guanylate cyclase, the major nitric oxide receptor, leads to moyamoya and achalasia. Am J Hum Genet 94:385-394, 2014

9. Hoshino H, Izawa Y, Suzuki N: Epidemiological features of moyamoya disease in Japan. Neurol Med Chir (Tokyo) 52:295-298, 2012

10. Ikeda H, Sasaki T, Yoshimoto T, Fukui M, Arinami T: Mapping of a familial moyamoya disease gene to chromosome 3p24.2-p26. Am J Hum Genet 64:533-537, 1999

11. Inoue TK, Ikezaki K, Sasazuki T, Matsushima T, Fukui M: Linkage analysis of moyamoya disease on chromosome 6 . J Child Neurol 15:179-182, 2000

12. Ito A, Fujimura M, Niizuma K, Kanoke A, Sakata H, MoritaFujimura Y, et al: Enhanced post-ischemic angiogenesis in mice lacking $R N F 213$; a susceptibility gene for moyamoya disease. Brain Res 1594:310-320, 2015

13. Kamada F, Aoki Y, Narisawa A, Abe Y, Komatsuzaki S, Kikuchi A, et al: A genome-wide association study identifies RNF213 as the first moyamoya disease gene. J Hum Genet 56:34-40, 2011

14. Kawaguchi S, Sakaki T, Morimoto T, Kakizaki T, Kamada $\mathrm{K}$ : Characteristics of intracranial aneurysms associated with moyamoya disease. A review of 111 cases. Acta Neurochir (Wien) 138:1287-1294, 1996 
15. Khan N, Achrol AS, Guzman R, Burns TC, Dodd R, BellStephens T, et al: Sex differences in clinical presentation and treatment outcomes in moyamoya disease. Neurosurgery 71:587-593, 2012

16. Kim SK, Cho BK, Phi JH, Lee JY, Chae JH, Kim KJ, et al: Pediatric moyamoya disease: An analysis of 410 consecutive cases. Ann Neurol 68:92-101, 2010

17. Kuroda S, Houkin K: Moyamoya disease: current concepts and future perspectives. Lancet Neurol 7:1056-1066, 2008

18. Liu W, Morito D, Takashima S, Mineharu Y, Kobayashi H, Hitomi T, et al: Identification of RNF213 as a susceptibility gene for moyamoya disease and its possible role in vascular development. PLoS One 6:e22542, 2011

19. Liu XJ, Zhang D, Wang S, Zhao YL, Teo M, Wang R, et al: Clinical features and long-term outcomes of moyamoya disease: a single-center experience with 528 cases in China. J Neurosurg 122:392-399, 2015

20. Metzker ML: Sequencing technologies - the next generation. Nat Rev Genet 11:31-46, 2010

21. Miao W, Zhao PL, Zhang YS, Liu HY, Chang Y, Ma J, et al: Epidemiological and clinical features of moyamoya disease in Nanjing, China. Clin Neurol Neurosurg 112:199-203, 2010

22. Miskinyte S, Butler MG, Hervé D, Sarret C, Nicolino M, Petralia JD, et al: Loss of BRCC3 deubiquitinating enzyme leads to abnormal angiogenesis and is associated with syndromic moyamoya. Am J Hum Genet 88:718-728, 2011

23. Miyatake S, Miyake N, Touho H, Nishimura-Tadaki A, Kondo Y, Okada I, et al: Homozygous c.14576G $>$ A variant of $R N F 213$ predicts early-onset and severe form of moyamoya disease. Neurology 78:803-810, 2012

24. Research Committee on the Pathology and Treatment of Spontaneous Occlusion of the Circle of Willis: Guidelines for diagnosis and treatment of moyamoya disease (spontaneous occlusion of the circle of Willis). Neurol Med Chir (Tokyo) 52:245-266, 2012

25. Sakurai K, Horiuchi Y, Ikeda H, Ikezaki K, Yoshimoto T, Fukui M, et al: A novel susceptibility locus for moyamoya disease on chromosome 8q23. J Hum Genet 49:278-281, 2004

26. Scott RM, Smith ER: Moyamoya disease and moyamoya syndrome. N Engl J Med 360:1226-1237, 2009

27. Suzuki J, Takaku A: Cerebrovascular "moyamoya" disease. Disease showing abnormal net-like vessels in base of brain. Arch Neurol 20:288-299, 1969
28. Tavira B, Gómez J, Santos F, Gil H, Alvarez V, Coto E: A labor- and cost-effective non-optical semiconductor (Ion Torrent) next-generation sequencing of the $S L C 12 A 3$ and $C L C N K A / B$ genes in Gitelman's syndrome patients. J Hum Genet 59:376-380, 2014

29. Wang X, Zhang Z, Liu W, Xiong Y, Sun W, Huang X, et al: Impacts and interactions of PDGFRB, MMP-3, TIMP-2, and $R N F 213$ polymorphisms on the risk of moyamoya disease in Han Chinese human subjects. Gene 526:437-442, 2013

30. Wu Z, Jiang H, Zhang L, Xu X, Zhang X, Kang Z, et al: Molecular analysis of $R N F 213$ gene for moyamoya disease in the Chinese Han population. PLoS One 7:e48179, 2012

\section{Disclosures}

The authors report no conflict of interest concerning the materials or methods used in this study or the findings specified in this paper.

\section{Author Contributions}

Conception and design: Zhao, X Zhang. Acquisition of data: Q Zhang, Y Liu, D Zhang, R Wang, F Liu, Zhou. Analysis and interpretation of data: Q Zhang, Y Liu, D Zhang, R Wang, Y Zhang, S Wang, Yu, Lu, F Liu. Drafting the article: Q Zhang, Y Liu. Critically revising the article: Q Zhang, Y Liu. Reviewed submitted version of manuscript: Q Zhang, Y Liu. Statistical analysis: Q Zhang, D Zhang, R Wang.

\section{Supplemental Information Online-Only Content}

Supplemental material is available with the online version of the article.

Supplemental Data. http://thejns.org/doi/suppl/10.3171/2016. 2.JNS152173.

\section{Correspondence}

Jizong Zhao, Department of Neurosurgery, Beijing Tiantan Hospital, Capital Medical University, China National Clinical Research Center for Neurological Diseases, 6 Tiantanxili, DongCheng District, Beijing 100050, China. email: zhaojz205@163.com. 